\title{
Personal Tour Planner
}

\author{
Mr. Abhishek Tapadia ${ }^{1}$, Mr. Kunal Agarwal ${ }^{2}$, Mr. Brijesh Sonawane ${ }^{3}$, \\ Mr. Shubham Akurdekar ${ }^{4}$, Prof. Mrs. Suchita Wankhade ${ }^{5}$ \\ Student, Comp Dept, Trinity College of Engineering and Research, Pune, India ${ }^{1,2,3,4}$ \\ Guide, Comp Dept, Trinity College of Engineering and Research, Pune, India ${ }^{5}$
}

\begin{abstract}
Nowadays, there are a number of online trip planning systems that make it possible to automatically generate a selection and routing plan for visiting Points of Interest (POIs) that satisfy the tourist personal interests. These systems implement various features and functionalities that aim to meet diverse tourist interest profiles. Some of the most important features that get more frequently utilized include tourist personal interest estimation, selection of POIs and path routing, mandatory POIs, dynamic recalculation of trip plan, multiple day trip planning, opening hours of POIs, budget limitations and weather dependency. In this paper, we make android application on personal travelling systems, by explaining their functionalities and features. In addition, we present an evaluation of a number of existing systems in respect to their level of adaption of the state of the art touristic trip planning features.
\end{abstract}

Keywords: Travelling, Tourist, Budget, Management, Geo-Fencing

\section{INTRODUCTION}

The main goal of a touristic trip planning system is to automatically plan the trip for visiting a number of Points of Interest (POI) in a travel destination. Thus, it would help the tourist to do the complicated task of planning individual visits, as well as preparation of trip itinerary. Moreover, the trip planners would take into account personal preferences of the tourist, as well as her/his context and (ideally) other influencing factors. The various apps are use for there specific purposes. And each app has its separate login id and passwords. These apps has different steps to use there services. User has to go through specific app for searching hotels, finding routs, booking cabs, searching places of user's demands. It will take time consuming activity, it also required more storage space. The proposed application will solved out the problem of storage and time by giving all this facilities together in one app itself. Suppose person visits to unfamiliar city. If he came to that city for educational purpose and for few days or months. He don't know about where to go?, how to go?, So Personal Travelling Scheduler will find out best places according to reviews and his interest. And also manages his budgets of how many days he will spends in this city.

\section{LITERATURE SURVEY}

"Smart Travel Planner: A mashup of travel-related web services", Rabia Jafri; Amal Saad Alkhunji, Ghada Khaled Alhader, Hanan Rabeiah Alrabeiah, Noura Abdullah Alhammad, Sara Khader Alzahrani, 2013 International Conference on Current Trends in Information Technology [1]

The Smart Travel Planner is a web-based intelligent system for facilitating the travel planning process, which is implemented as a mashup of several travel-related services' APIs. Unlike other similar applications which essentially work as calendaring programs, our system is unique in that it integrates the information and functions needed for travel planning in their entirety within a single piece of software providing such features as checking hotel availability and calculating optimum routes between user-specified locations at various travel destinations, and offering suggestions for sightseeing, all the while making sure to take into consideration the user-specified needs for particular meeting days, times and locations and personal preferences for dining, relaxation and sleep. Furthermore, these services are not limited to a particular city but are offered for any city around the world for which such information exists within the databases of the services included in the mashup.

" ANALOC: Efficient analytics over Location Based Services " Md Farhadur Rahman, Saad Bin Suhaim, Weimo Liu, Saravanan Thirumuruganathan, Nan Zhang, Gautam Das [2]

ANALOC, a prototypical system for enabling the analytics of data underlying real-world Location Based Services (LBS) by using nothing but the K Nearest Neighbor (knn) search interface (often web-based) publicly provided by the LBS. Location Based Services (LBS), including standalone ones such as Google Maps and embedded ones such as 
Vol. 8, Issue 4, April 2019

"users near me" in the we chat instant-messaging platform, provide great utility to millions of users. Unfortunately, it is not easy to tap into these LBS for tasks such as data analytics and mining, because the only access interface they offer is a limited k-Nearest-Neighbor $(\mathrm{kNN})$ search interface - i.e., for a given input location, return the $\mathrm{k}$ nearest tuples in the database, where $\mathrm{k}$ is a small constant such as 50 or 100 .

"Proposal of Multiple Travel Scheduling System based on Inverse Operation Method", Kazuya Murata, Takayuki Fujimoto, shed in: 2015 IEEE/ACIS 14th International Conference on Computer and Information Science[3]

Currently, a tourist and travellers of a foreigner coming in Japan increase. "Tokyo Olympics" are holds in 2020, and the increase in tourist and traveller is anticipated from now on. As a main sightseeing method, many tourists and travellers check a famous sightseeing spot beforehand and come to Japan. In addition, in the late years, as well as a famous sightseeing spot, tourists and travellers want to know the local charm. Furthermore, sightseeing information and map application system of the Internet develop rapidly. For reasons of them, a tourist and traveller of a foreigner enjoying a great variety of sightseeing are in a tendency to increase. But, in the inexperienced destination, a tourist and traveller do not often know sojourn time in the sightseeing spot and movement using a public institution, and it is the present conditions that such sightseeing and trip are difficult. Particularly, tourist and travellers has the prescribed time that departure time of train and check-in and check-out of the hotel. Because it is necessary to keep the prescribed time, it is the present conditions to have difficult inexperienced movement. Therefore we thought about a new form of sightseeing application system. In this system, we set present place, return place, planned time to return and sightseeing categories. By this setting, we propose the system that it can automatically show a sightseeing route and sightseeing plan in set time.

\section{PROPOSE SYSTEM}

In a proposed system we are making android application.

In which User will give an Input like place. By Using shortest path, to Detect near place of a park Then with the help of shortest path and a Name of a place. Finally, User will Get nearby place whish w/ she they want to go. The system will display places to the tourist according wishes to his interest and budget within particular distance. And collect information of different places at one place with the help of Local Guide .System will analyze the user type based on his interest To manage the money according to user interest. To manage the storage space on the device

\section{SYSTEM ARCHITECTURE}

Following diagram is our system's architecture diagram:
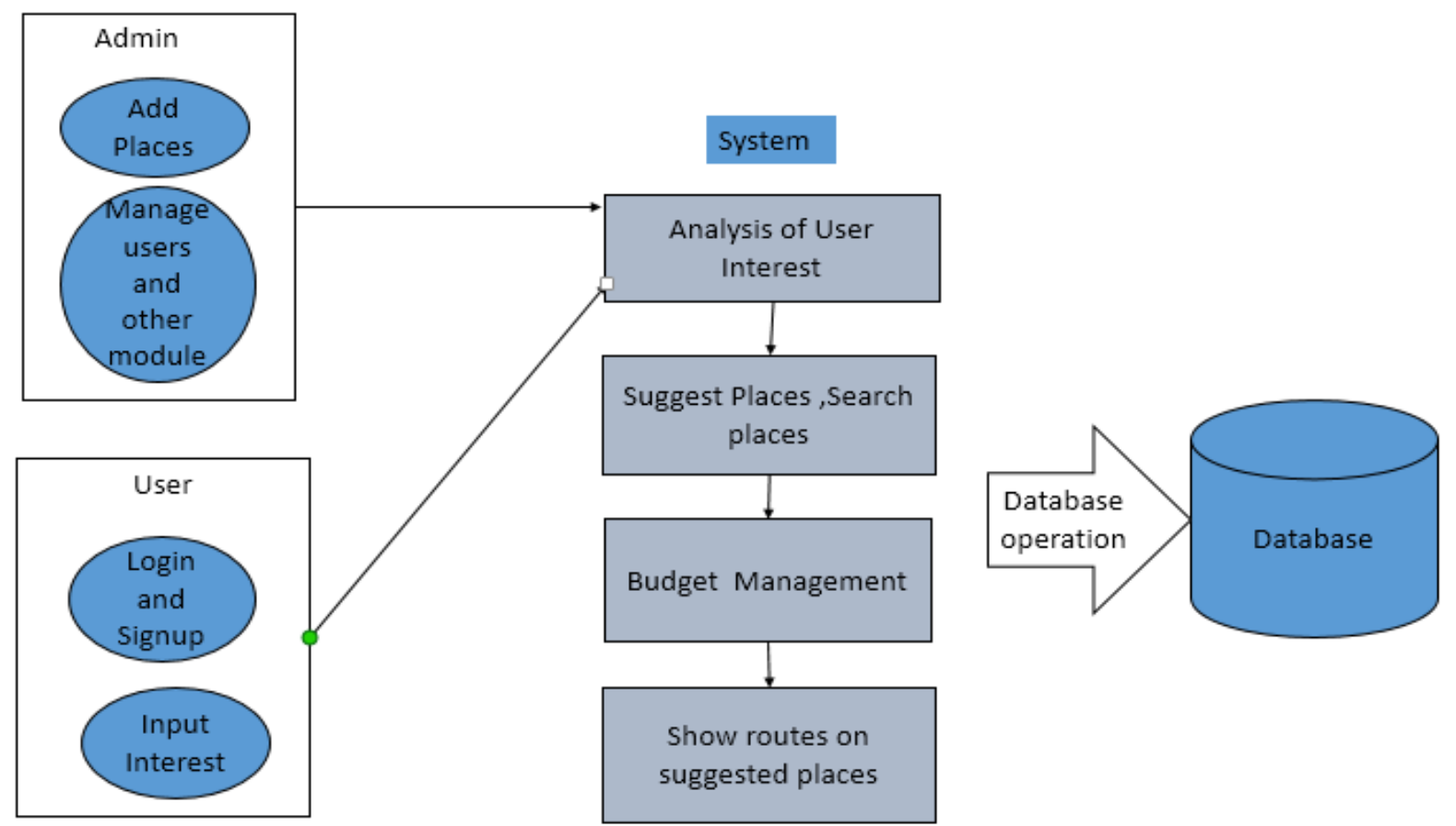

Figure 1: system architecture 


\section{International Journal of Advanced Research in Computer and Communication Engineering}

Vol. 8, Issue 4, April 2019

- User Module:-In user module user have to get registered with system with unique id and password. After successfully registeration user can login with system.

- Login:-After successful registeration ,user can login and get authority to access system of personal travelling scheduler.

- $\quad$ Input Interest and budget.:-When user get login successfully ,user access operation like input interest and budget and enter their interest and budget as he/she want.

- $\quad$ Search places:-User can search the different places in want to travel on his budget.

- $\quad$ Rate and review the places.:-After searching places he/she want depend on his budget then user can get give rate and review to places

- $\quad$ Administrator module:-Admin module has responsibility of adding user and managing other modules

- $\quad$ Add different places and Information:-We can add different places we want.

\section{METHODOLOGIES}

\section{Algorithm Geo Fencing}

Typically geofencing is thought as something that only works outdoors, utilizing GPS technology. But actually, you can use it with any technology. Depending on your use case and the accuracy you need for it, you could utilize a range of positioning technologies, such as: Wi-fi, GPS, cellular data, geomagnetic and Bluetooth beacons. What technology or technologies to use, depends a lot on where the geofencing will happen. In outdoors for larger geofences you could get good results with cellular and WiFi data, which gives better battery efficiency than GPS. Geo Fencing algorithm is use to find out nearby location of user interest.

\section{Steps}

1. To make use of geofencing, an administrator or developer must first establish a virtual boundary around a specified location in GPS- or RFID-enabled software.

2. This can be as simple as a circle drawn 100 feet around a location on Google Maps, as specified using APIs when developing a mobile app.

3. This virtual geofence will then trigger a response when an authorized device enters or exits that area, as specified by the administrator or developer.

4. A geofence is most commonly defined within the code of a mobile application, especially since users need to opt-in to location services for the geofence to work.

\section{Geo Fencing}

PolyGon myRoute = new PolyGon(points);

\section{bool stat = myRoute.FindPoint $($ Double.Parse $($ txtLat.Text.ToString ()$)$,} Double.Parse(txtLang.Text.ToString()));

\section{if(stat)}

\{

lblResult.Text = "Point found in the route";

\}

else

lblResult.Text = "Point not found in the route";

\section{CONCLUSION}

This is an android application that can reduce the efforts of the user as compare to the other application and it provide the following features like Money Management, Display places to the tourist according wishes to his interest and budget within particular distance, Manage the storage space on the device. 


\section{FUTURE WORK}

- This systems are truly helpful when the recommended item is something that the user has not seen in the past.

- We are providing registration and login credentials to users for this application.

- In order to develop this kind of methodology mobility system we consider both active (planning) and passive (monitoring) roles for GIS, as an interactive technology that can use geographical information to help provide solutions to a range of issues facilitating territorial process.

The technological upgrade in integrated and intermodal mobility and transportation services, a few prototypes of vehicle and micro-grid will be realized, that will be extensively tested and demonstrated

\section{REFERRENCES}

[1]. Smart Travel Planner: A mashup of travel-related web services”, Rabia Jafri ; Amal Saad Alkhunji ,Ghada Khaled Alhader, Hanan Rabeiah Alrabeiah, Noura Abdullah Alhammad, Sara Khader Alzahrani,2013 International Conference on Current Trends in Information Technology]

[2]. ANALOC: Efficient analytics over Location Based Services “ Md Farhadur Rahman, Saad Bin Suhaim, Weimo Liu, Saravanan Thirumuruganathan, Nan Zhang, Gautam Das

[3]. Proposal of Multiple Travel Scheduling System based on Inverse Operation Method", Kazuya Murata ,Takayuki Fujimoto, shed in: 2015 IEEE/ACIS 14th International Conference on Computer and Information Science

[4]. "M. Wernke, P. Skvortsov, F. Durr, and K. Rothermel, "A classification" of location privacy attacks and approaches ," Personal and Ubiquitous Computing, vol. 18, no. 1, pp. 163-175, 2014.

[5]. B. Niu, Q. Li, X. Zhu, G. Cao, and H. Li, “Achieving k-anonymity in privacyaware location-based services," in IEEE INFOCOM, 2014.

[6]. M. E. Andres, N. E. Bordenabe, K. Chatzikokolakis, and C. Palamidessi, "Geo-in distinguish ability: Differential privacy for location-based systems," in ACM CCS, 2013 